T. Komatsu and S. Nakano

Nagoya Math. J.

Vol. 164 (2001), 133-146

\title{
ON THE GALOIS MODULE STRUCTURE OF IDEAL CLASS GROUPS
}

\author{
TORU KOMATSU AND SHIN NAKANO
}

\begin{abstract}
Let $K / k$ be a Galois extension of a number field of degree $n$ and $p$ a prime number which does not divide $n$. The study of the $p$-rank of the ideal class group of $K$ by using those of intermediate fields of $K / k$ has been made by Iwasawa, Masley et al., attaining the results obtained under respective constraining assumptions. In the present paper we shall show that we can remove these assumptions, and give more general results under a unified viewpoint. Finally, we shall add a remark on the class numbers of cyclic extensions of prime degree of $\mathbb{Q}$.
\end{abstract}

\section{Introduction}

Throughout this paper, $p$ will denote a prime number and $\mathbb{F}_{p}$ the finite field with $p$ elements. For an integer $n>1$ prime to $p$, denote by $c(n, p)$ the order of $p$ in the multiplicative group $(\mathbb{Z} / n \mathbb{Z})^{\times}$, and by $d(n, p)$ the minimum of $c(l, p)$ for all prime factors $l$ of $n$. For a finite abelian group $A$, denote by $r_{p} A$ its $p$-rank; $r_{p} A=\operatorname{dim}_{\mathbb{F}_{p}}(A / p A)$. We will use this notation $r_{p}$ instead of $\operatorname{dim}_{\mathbb{F}_{p}}$ even for vector spaces over $\mathbb{F}_{p}$. Denote by $C(K)$ and $h(K)$ the ideal class group and the class number of an algebraic number field $K$.

Let $k$ be an algebraic number field of finite degree and $K$ a Galois extension of $k$ of degree $n$ prime to $p$. Iwasawa [5] deduced the inequality $r_{p} C(K) \geqq d(n, p)$ under the conditions $p \nmid h(k)$ and $p \mid h(K)$ from the following group theoretical proposition:

Let $G$ be a group of order $n$ prime to $p$ and $M$ an $\mathbb{F}_{p}[G]$-module such that the action of $G$ on $M$ is non-trivial. Then $r_{p} M \geq$ $d(n, p)$.

Suppose $G$ and $M$ are as above. Then $G$ acts non-trivially on the quotient module $M / M^{G}$, where $M^{G}$ is the submodule of $M$ consisting of elements

Received May 26, 2000.

Revised September 22, 2000.

2000 Mathematics Subject Classification: 11R29. 
left fixed by $G$. Therefore the conclusion of the above property can be replaced by $r_{p} M-r_{p} M^{G} \geq d(n, p)$. From this simple observation, we can improve a little the above result of Iwasawa on ideal class groups. It will be seen that $M / M^{G}$ is isomorphic to the kernel of the norm map: $M \rightarrow M$, $x \mapsto \sum_{\sigma \in G} \sigma x$.

In this paper, we shall utilize the kernels of the maps of this kind to study the behavior of the $p$-rank of finite $G$-modules, and apply it to the rank of ideal class groups. After group theoretical discussions of same kind as above, we shall apply them to the estimation of the rank of ideal class groups, and extend the results of Masley[7], Cornell-Rosen[3] and others, as well as to the non- $l$-part of the ideal class group in $\mathbb{Z}_{l}$-extensions. Finally, we shall add a remark on the class numbers of cyclic fields of prime degrees of $\mathbb{Q}$ including a conjecture.

The authors would like to thank the referee for valuable suggestions.

\section{$\S 1$. Group theoretical discussions}

Let $G$ be a finite group and $M$ a finite $G$-module. Let $x \in M$. The stabilizer of $x$ is denoted by $G_{x}$ and the $G$-orbit of $x$ by $G x$, that is,

$$
G_{x}=\{\sigma \in G \mid \sigma x=x\}, \quad G x=\{\sigma x \in M \mid \sigma \in G\}
$$

It is familiar that $|G x|=\left(G: G_{x}\right)$.

LEMmA 1. Assume that $G_{x}=\{1\}$ for every $x(\neq 0) \in M$. Then we have $|M| \equiv 1(\bmod |G|)$.

Proof. Decompose $M$ to the $G$-orbits: $M=\{0\} \cup G x_{1} \cup \cdots \cup G x_{t}$. It follows from the assumption that $\left|G x_{i}\right|=\left(G: G_{x_{i}}\right)=|G|(1 \leq i \leq t)$. Hence we have $|M|=1+t|G| \equiv 1(\bmod |G|)$.

For a subgroup $H$ of $G$, we define a subset $M^{H}$ of $M$ by

$$
M^{H}=\{x \in M \mid \sigma x=x \text { for all } \sigma \in H\} .
$$

The norm map $N_{H}$ for $H$ is an endomorphism of $M$ as an $H$-module defined by

$$
N_{H}: M \rightarrow M, \quad x \mapsto \sum_{\sigma \in H} \sigma x
$$


Put $H(\sigma)=\sigma H \sigma^{-1}$ for $\sigma \in G$. We easily see that

$$
\sigma M^{H}=M^{H(\sigma)}, \quad \sigma N_{H} x=N_{H(\sigma)}(\sigma x)
$$

for $\sigma \in G$ and $x \in M$. In particular, if $H$ is a normal subgroup of $G$ then $M^{H}$ is a $G$-module and $N_{H}$ is a $G$-homomorphism.

LEMMA 2. Let $H$ be a subgroup of $G$.

(1) $\operatorname{Ker} N_{H} \subseteq \operatorname{Ker} N_{G}$.

(2) If $H$ is a normal subgroup acting on $M$ trivially, then $M$ becomes a $G / H$-module and $\operatorname{Ker} N_{G / H} \subseteq \operatorname{Ker} N_{G}$.

Proof. Use the decomposition to the cosets of $H$ in $G$.

LEMmA 3. If $\left(|G|,\left|M^{G}\right|\right)=1$, then $N_{G} M=M^{G}$ and $M=M^{G} \oplus$ $\operatorname{Ker} N_{G}$.

Proof. The inclusion $N_{G} M \subseteq M^{G}$ is clear. We must make sure of the converse. Let $x \in M^{G}$. Then we have $N_{G} x=|G| x$. It follows from the assumption that there exists an $a \in \mathbb{Z}$ such that $a|G| \equiv 1\left(\bmod \left|M^{G}\right|\right)$. Thus $x=a|G| x=a N_{G} x$ and we conclude $M^{G} \subseteq N_{G} M$. Furthermore, the same argument shows that $M^{G} \cap \operatorname{Ker} N_{G}=\{0\}$. Comparing the orders, we obtain $M=M^{G} \oplus \operatorname{Ker} N_{G}$.

Lemma 4. If $|G|$ is prime and $\left(|G|,\left|\operatorname{Ker} N_{G}\right|\right)=1$, then $\left|\operatorname{Ker} N_{G}\right| \equiv 1$ $(\bmod |G|)$.

Proof. Let $x(\neq 0) \in \operatorname{Ker} N_{G}$. If $G_{x}=G$, then $0=N_{G} x=|G| x$ which yields $x=0$ by the assumption $\left(|G|,\left|\operatorname{Ker} N_{G}\right|\right)=1$. This is a contradiction. Thus we have $G_{x} \subsetneq G$. Since the order of $G$ is prime, we have $G_{x}=\{1\}$ which completes the proof by Lemma 1.

In order to state our results on the $p$-rank of $G$-modules, we shall use the notation $c(n, p), d(n, p)$ defined in Introduction for an integer $n>1$ prime to $p$, and one more notation $e(n, p)$ defined as the greatest common divisor of $c(l, p)$ for all prime factors $l$ of $n$. The following relations hold among them: 


$$
\begin{gathered}
c(l, p) \leq c(n, p) \\
\| \\
d(n, p) \leq d(l, p) \\
\vee\|\quad\| \\
e(n, p) \leq e(l, p)
\end{gathered}
$$

where $l$ is a prime factor of $n$. For an abelian group $A$ and a positive integer $m$, let $A[m]$ be the kernel of the multiplication-by- $m$ map, i.e.,

$$
A[m]=\{a \in A \mid m a=0\} .
$$

It is easy to see that $A[p]$ is an $\mathbb{F}_{p}$-vector space and its dimension is equal to $r_{p} A$ provided $A$ is finite.

Proposition 1. Let $G$ be a cyclic group of prime order $l \neq p$ and $M$ a finite $G$-module. Then we have

$$
r_{p} \operatorname{Ker} N_{G} \equiv 0 \quad(\bmod c(l, p)) .
$$

Proof. One may easily check that $r_{p} \operatorname{Ker} N_{G}=r_{p}\left(M[p] \cap \operatorname{Ker} N_{G}\right)$. So we can assume $M$ to be an $\mathbb{F}_{p}$-space. Then, by Lemma 4 , we have $\left|\operatorname{Ker} N_{G}\right|=p^{r_{p} \operatorname{Ker} N_{G}} \equiv 1(\bmod l)$ which implies the desired congruence from the definition of $c(l, p)$.

Remark. We have $r_{p} M=r_{p} M^{G}+r_{p} \operatorname{Ker} N_{G}$ by Lemma 3. So the conclusion of the above proposition can be replaced by

$$
r_{p} M-r_{p} M^{G} \equiv 0 \quad(\bmod c(l, p)),
$$

like in the following results (Propositions 2 and 3).

We next state two propositions which extend slightly the results of Iwasawa [5], Cornell-Rosen [3] or Cornell [2]. Though the heart of the proof may be found in their original arguments, we present here an approach via the direct use of the kernels of norm maps, which seems to us more easily comprehensible.

Proposition 2. Let $G$ be a group of order $n$ prime to $p$. Let $M$ be a finite p-group and also a $G$-module on which the action of $G$ is non-trivial. Then we have

$$
r_{p} \operatorname{Ker} N_{G} \geq d(n, p)
$$


Proof. Choose $\sigma \in G$ with minimal order such that the action of $\sigma$ on $M$ is non-trivial. Let $l$ be a prime dividing the order of $\sigma$. Put $H=\langle\sigma\rangle /\left\langle\sigma^{l}\right\rangle$. Since $\sigma^{l}$ acts on $M$ trivially, $M$ is an $H$-module and the action of $H$ on $M$ is non-trivial. (The use of such $H$ is owing to Iwasawa [5].) One can use Proposition 1 to see $r_{p} \operatorname{Ker} N_{H} \equiv 0(\bmod c(l, p))$. If $\operatorname{Ker} N_{H}=\{0\}$ then the norm map $N_{H}$ is injective and consequently $M=N_{H} M \subseteq M^{H} \subseteq M$. This means $M=M^{H}$ which contradicts the non-triviality of the action of $H$. Thus we conclude $\operatorname{Ker} N_{H} \neq\{0\}$. It follows from Lemma 2 that $\operatorname{Ker} N_{H} \subseteq \operatorname{Ker} N_{\langle\sigma\rangle} \subseteq \operatorname{Ker} N_{G}$. Hence

$$
r_{p} \operatorname{Ker} N_{G} \geq r_{p} \operatorname{Ker} N_{H} \geq c(l, p) \geq d(n, p),
$$

which completes the proof.

Proposition 3. Let $G$ be a solvable group of order $n$ prime to $p$ and $M$ a finite $G$-module. Then there exists a non-negative integer $x_{l}$ for each prime factor $l$ of $n$ such that

$$
r_{p} \operatorname{Ker} N_{G}=\sum_{l \mid n} x_{l} c(l, p)
$$

therefore we have

$$
r_{p} \operatorname{Ker} N_{G} \equiv 0 \quad(\bmod e(n, p)) .
$$

Proof. Since $G$ is solvable, $G$ has the composition series

$$
G=G_{0} \supset G_{1} \supset \cdots \supset G_{t}=\{1\}
$$

such that every factor group $H_{i}=G_{i-1} / G_{i}$ is cyclic of prime order. In view of the sequence

$$
M^{G}=M^{G_{0}} \subseteq M^{G_{1}} \subseteq \cdots \subseteq M^{G_{t}}=M
$$

we write

$$
r_{p} M-r_{p} M^{G}=\sum_{i=1}^{t}\left(r_{p} M^{G_{i}}-r_{p} M^{G_{i-1}}\right) .
$$

Note that $M^{G_{i}}$ is an $H_{i}$-module and further $\left(M^{G_{i}}\right)^{H_{i}}=M^{G_{i-1}}$. Therefore, by Lemma 3 and Proposition 1 ,

$$
r_{p} M^{G_{i}}-r_{p} M^{G_{i-1}}=r_{p} \operatorname{Ker}\left(N_{H_{i}}: M^{G_{i}} \rightarrow M^{G_{i}}\right) \equiv 0 \quad\left(\bmod c\left(l_{i}, p\right)\right),
$$


where $l_{i}=\left|H_{i}\right|=\left(G_{i-1}: G_{i}\right)$. Hence we can take non-negative integers $y_{i}$ such that

$$
r_{p} \operatorname{Ker} N_{G}=r_{p} M-r_{p} M^{G}=\sum_{i=1}^{t} y_{i} c\left(l_{i}, p\right) .
$$

This proves our assertion.

One may notice that Proposition 3 includes Proposition 1. In the rest of this section, we will extend Proposition 1 in a different direction. Define a subset $\Gamma_{G} M$ of $M$ by

$$
\Gamma_{G} M=\bigcap_{H} \operatorname{Ker}\left(N_{H}: M \rightarrow M\right)
$$

where $H$ runs through all the subgroups of $G$ such that $H \neq\{1\}$. Here, even if the running range of $H$ is restricted to all the cyclic subgroups of prime order, we have the same set as in the right-hand side of the above formula. This is confirmed by the fact that if subgroups $H_{1}, H_{2}$ satisfy $H_{1} \subseteq H_{2}$ then $\operatorname{Ker} N_{H_{1}} \subseteq \operatorname{Ker} N_{H_{2}}$ by Lemma 2. It must be also noted that $\Gamma_{G} M$ becomes a $G$-module, as shown by the relation $\sigma \operatorname{Ker} N_{H}=\operatorname{Ker} N_{H(\sigma)}$ for $\sigma \in G$.

LEMmA 5. If $\left(|G|,\left|\Gamma_{G} M\right|\right)=1$, then $\left|\Gamma_{G} M\right| \equiv 1(\bmod |G|)$.

Proof. Let $x$ be a non-zero element of $\Gamma_{G} M$. From Lemma 1, it is enough to show that $G_{x}=\{1\}$. Suppose that there exists $\sigma(\neq 1) \in G_{x}$. Let $s$ be the order of $\sigma$. It follows from $x \in \Gamma_{G} M \subseteq \operatorname{Ker} N_{\langle\sigma\rangle}$ that $s x=N_{\langle\sigma\rangle} x=0$; thus we have $x=0$ by the assumption. This is a contradiction.

The next proposition is shown in the same way as Proposition 1 using this lemma.

Proposition 4. Let $G$ be a group of order $n$ prime to $p$ and $M a$ finite $G$-module. Then we have

$$
r_{p} \Gamma_{G} M \equiv 0 \quad(\bmod c(n, p))
$$

This is a natural generalization of Proposition 1 , because $\Gamma_{G} M$ is nothing but $\operatorname{Ker} N_{G}$ in case $G$ is cyclic of prime order. 


\section{$\S 2$. Rank of ideal class groups}

Throughout this section, $k$ denotes a finite algebraic number field. We will apply the results of the previous section to the $p$-rank of the ideal class groups. We shall describe the behavior of $p$-rank for a Galois extension of $k$.

Let $K / k$ be a Galois extension of degree $n$ with the Galois group $G$. Note that both $C(K)$ and $C(K)[p]$ are $G$-modules. Furthermore, we can show that if $p$ does not divide $n$ then $C(k)[p]$ is naturally isomorphic to $C(K)[p]^{G}=C(K)^{G}[p]$. Thus, for the usual norm map $N_{K / k}: C(K) \rightarrow$ $C(k)$, we have

$$
r_{p} \operatorname{Ker} N_{K / k}=r_{p} C(K)-r_{p} C(K)^{G}=r_{p} C(K)-r_{p} C(k) .
$$

From this, we obtain Theorems 1, 2, 3 below as consequence of Propositions 1, 2 and 3 , respectively. We add that Theorems 2 and 3 improve the results of [5], [3] or [2].

TheOREm 1. Let $K / k$ be a cyclic extension of prime degree $l \neq p$. Then we have

$$
r_{p} C(K) \equiv r_{p} C(k) \quad(\bmod c(l, p)) .
$$

Theorem 2. Let $K / k$ be a Galois extension of degree $n$ prime to $p$. Assume that $r_{p} C(K) \neq r_{p} C(k)$. Then we have

$$
r_{p} C(K)-r_{p} C(k) \geq d(n, p) .
$$

TheOREm 3. Let $K / k$ be a solvable extension of degree $n$ prime to $p$. Then there exists a non-negative integer $x_{l}$ for each prime factor $l$ of $n$ such that

$$
r_{p} C(K)-r_{p} C(k)=\sum_{l \mid n} x_{l} c(l, p)
$$

therefore we have

$$
r_{p} C(K) \equiv r_{p} C(k) \quad(\bmod e(n, p)) .
$$

Next, in order to restate Proposition 4, we define a subgroup $B(K / k)$ of $C(K)$, for a finite Galois extension $K / k$, by

$$
B(K / k)=\bigcap_{F} \operatorname{Ker}\left(N_{K / F}: C(K) \rightarrow C(F)\right),
$$


where $F$ runs through all the fields such that $k \subseteq F \subsetneq K$ and $K / F$ is cyclic of prime degree. Though we may make $F$ run through all the intermediate fields $(\neq K)$, the right-hand side turns out to be the same group as above, as remarked after the definition of $\Gamma_{G} M$. Then Proposition 4 shows the following general result.

THEOREM 4. Let $K / k$ be a Galois extension of degree $n$ prime to $p$. Then we have

$$
r_{p} B(K / k) \equiv 0 \quad(\bmod c(n, p))
$$

There are several corollaries of Theorem 4. We first present the result on a cyclic extension which generalizes Theorem 1 naturally.

Corollary 1. Let $K / k$ be a cyclic extension of prime degree $l \neq p$. Assume that there is a subfield $k_{0}$ of $k$ such that $K / k_{0}$ is cyclic of degree $l^{d}$. Then we have

$$
r_{p} C(K) \equiv r_{p} C(k) \quad\left(\bmod c\left(l^{d}, p\right)\right)
$$

Proof. Use the identity $B\left(K / k_{0}\right)=\operatorname{Ker} N_{K / k}$.

This leads us to the result on the non- $l$-part of the ideal class groups in a $\mathbb{Z}_{l}$-extension.

COROLlary 2. Let $l$ be a prime different from $p$. Let $k_{\infty} / k$ be a $\mathbb{Z}_{l^{-}}$ extension and $k_{n}$ its $n$-th layer, that $i s,\left[k_{n}: k\right]=l^{n}$. Then we have

$$
r_{p} C\left(k_{n}\right) \equiv r_{p} C\left(k_{n-1}\right) \quad\left(\bmod c\left(l^{n}, p\right)\right),
$$

for $n \geq 1$.

Remark. If $k$ is abelian over $\mathbb{Q}$, then there is no interest in applying this result to the layers of sufficiently large degrees. In fact, Washington [9] has proved that, in such a case, the non-l-part of the class number $h\left(k_{n}\right)$ is bounded as $n \rightarrow \infty$ (see also [10, Ch. 16]).

Next we generalize the result of Masley [7] where the restricted case that $K / k$ is abelian and moreover $r_{p} C(k)=0$ is treated (cf. [3] and [10, Ch. 10]). 
Corollary 3. Let $K / k$ be a Galois extension of degree $n$ prime to $p$. Assume that $r_{p} C(F)=r_{p} C(k)$ for every intermediate fields $k \subseteq F \subsetneq K$ such that $K / F$ is cyclic of prime degree. Then we have

$$
r_{p} C(K) \equiv r_{p} C(k) \quad(\bmod c(n, p)) .
$$

Proof. We abbreviate $\left(\operatorname{Ker} N_{K / F}\right)[p]$ to $D(F)$ for an intermediate field $F$ of $K / k$. Then we have $r_{p} D(F)=r_{p} \operatorname{Ker} N_{K / F}=r_{p} C(K)-r_{p} C(F)$. If $K / F$ is cyclic of prime degree, then $r_{p} D(F)=r_{p} D(k)$ by our assumption, and thus $D(F)=D(k)$. Hence we find $B(K / k)[p]=D(k)$. The desired congruence follows from this and Theorem 4.

Remark. Let $A$ be a finite abelian group and $m$ a positive integer. We define $p^{m}$-rank of $A$ by $r_{p^{m}} A=\operatorname{dim}_{\mathbb{F}_{p}}\left(p^{m-1} A / p^{m} A\right)$. Obviously, it is also given by $r_{p^{m}} A=r_{p}\left(A\left[p^{m}\right] / A\left[p^{m-1}\right]\right)$. Considering $C(K)\left[p^{m}\right] / C(K)\left[p^{m-1}\right]$ instead of $C(K)[p]$, we may extend all the results of this section to those on the $p^{m}$-rank of ideal class groups. For example, Theorem 2 is generalized as

$$
r_{p^{m}} C(K)-r_{p^{m}} C(k) \geq d(n, p) \text {, if } r_{p^{m}} C(K) \neq r_{p^{m}} C(k),
$$

and the conclusion of Theorem 4 is rewritten in the form

$$
r_{p^{m}} B(K / k) \equiv 0 \quad(\bmod c(n, p)),
$$

for a positive integer $m$. The other results are also to be modified in the same manner. Note that $c(n, p)$ is independent of $m$.

\section{$\S 3$. A remark on cyclic extensions of prime degrees of $\mathbb{Q}$}

The results mentioned in the previous section are of a nature to restrict the structure of the ideal class group $C(K)$ of a number field $K$ by those of its subfields. We now consider the simplest case where $K$ is a cyclic extension over $\mathbb{Q}$ of prime degree $l$. The set of the class numbers $h(K)$ of all such fields $K$ is denoted by $H(l)$. We define a set $\mathcal{H}(l)$ of positive integers by

$$
\mathcal{H}(l)=\left\{a \in \mathbb{N} \mid p^{v_{p}(a)} \equiv 1(\bmod l) \text { for all prime numbers } p \neq l\right\},
$$

where $v_{p}(a)$ is the additive $p$-adic valuation of $a$, that is, the non-negative integer $v$ satisfying $p^{v} \mid a$ and $p^{v+1} \backslash a$.

Proposition 5. For a prime $l$, we have $H(l) \subset \mathcal{H}(l)$. 
Proof. Let $h \in H(l)$ and take an arbitrary cyclic extension $K$ over $\mathbb{Q}$ of degree $l$ with $h=h(K)$. Then for any power $p^{m}$ of a prime $\neq l$, we find $r_{p^{m}} C(K) \equiv 0(\bmod c(l, p))$ by the " $p^{m}$-rank version" of Theorem 1 . Thus

$$
v_{p}(h)=\sum_{m=1}^{\infty} r_{p^{m}} C(K) \equiv 0 \quad(\bmod c(l, p)),
$$

which means $p^{v_{p}(h)} \equiv 1(\bmod l)$. Hence $h \in \mathcal{H}(l)$.

It seems to us that the converse inclusion is also true, and we would like to propose the following

\section{Conjecture 1. $H(l)=\mathcal{H}(l)$.}

Since $\mathcal{H}(2)=\mathbb{N}$, the conjecture for $l=2$ says that there exists a quadratic field of which the class number is any given positive integer.

Put $H_{x}(l)=\{h \in H(l) \mid h \leq x\}$ and define $\mathcal{H}_{x}(l)$ similarly. We have carried out a computer search whether $H_{x}(l)=\mathcal{H}_{x}(l)$ or not for $l=2,3,5$ and for several values of $x$. Using PARI/GP and KASH, we could confirm the following;

$$
H_{5000}(2)=\mathcal{H}_{5000}(2), \quad H_{283}(3)=\mathcal{H}_{283}(3), \quad H_{81}(5)=\mathcal{H}_{81}(5) .
$$

We will illustrate them with some numerical results. First, we have made the complete list of the class numbers of quadratic fields $\mathbb{Q}(\sqrt{-m})$ with positive square-free integers $m$ up to $10^{7}$. Any positive integer $h \leq 5000$ appears in the list as a class number except $h=4801$ and 4921. A little further search revealed $h(\mathbb{Q}(\sqrt{-10074671}))=4801$ and $h(\mathbb{Q}(\sqrt{-10483871}))=4921$. Next, to examine the case of the cyclic cubic fields, we have used the polynomial $F_{m}(X)=X^{3}+m X^{2}-(m+3) X+1$ with $m \in \mathbb{Q}$ that parameterizes all the cyclic cubic extensions of $\mathbb{Q}$ (cf. [8]). The computer search showed that all integers $h \in \mathcal{H}_{350}(3) \backslash\{289,337\}$ are covered with the class numbers of the cubic fields $K_{m}$ defined by $F_{m}(X)=0$ for irreducible fractions $m=s / r$ where $1 \leq r, s \leq 1000$. In Tables 1 and 2 we present $K_{m}$ of class number $h$ which has the least conductor $\mathfrak{f}$ in the running range of $m=s / r$. In quintic case, several types of parametric polynomials with cyclic Galois group of degree 5 appear in [1, Ch. 5], [4], [6] and others. We could use them to discover a cyclic quintic field of class number $h$ for each $h \in \mathcal{H}(5)$ less than 200 with three exceptions. Table 3 shows irreducible polynomials $g(X)$ that define such fields and their conductors $\mathfrak{f}$. 
Table 1: Cyclic Cubic Fields

\begin{tabular}{|c|c|c|c|c|c|}
\hline$h$ & $m$ & $\mathfrak{f}$ & $h$ & $m$ & $\mathfrak{f}$ \\
\hline 1 & $5 / 1$ & 7 & 75 & $59 / 12$ & 6901 \\
\hline 3 & $3 / 2$ & 63 & 76 & $163 / 1$ & 27067 \\
\hline 4 & $11 / 1$ & 163 & 79 & $146 / 103$ & 161911 \\
\hline 7 & $16 / 1$ & 313 & 81 & $73 / 8$ & 7657 \\
\hline 9 & $3 / 8$ & 657 & 84 & $37 / 18$ & 6283 \\
\hline 12 & $31 / 16$ & 679 & 91 & $205 / 1$ & 42649 \\
\hline 13 & $31 / 1$ & 1063 & 93 & $116 / 1$ & 13813 \\
\hline 16 & $195 / 14$ & 1777 & 97 & $77 / 122$ & 168067 \\
\hline 19 & $37 / 1$ & 1489 & 100 & $136 / 1$ & 18913 \\
\hline 21 & $111 / 34$ & 1261 & 103 & $139 / 6$ & 22147 \\
\hline 25 & $345 / 58$ & 7753 & 108 & $126 / 1$ & 16263 \\
\hline 27 & $47 / 8$ & 3913 & 109 & $89 / 10$ & 11491 \\
\hline 28 & $336 / 1$ & 4219 & 111 & $114 / 1$ & 13347 \\
\hline 31 & $70 / 1$ & 5119 & 112 & $142 / 1$ & 20599 \\
\hline 36 & $27 / 4$ & 1197 & 117 & $156 / 1$ & 8271 \\
\hline 37 & $471 / 1$ & 8269 & 121 & $239 / 1$ & 57847 \\
\hline 39 & $29 / 6$ & 1687 & 124 & $284 / 1$ & 81517 \\
\hline 43 & $107 / 1$ & 11779 & 127 & $121 / 1$ & 15013 \\
\hline 48 & $9 / 16$ & 2817 & 129 & $407 / 351$ & 17557 \\
\hline 49 & $444 / 1$ & 7351 & 133 & $200 / 1$ & 40609 \\
\hline 52 & $127 / 1$ & 16519 & 139 & $322 / 1$ & 104659 \\
\hline 57 & $23 / 18$ & 4687 & 144 & $127 / 153$ & 21931 \\
\hline 61 & $86 / 15$ & 13291 & 147 & $31 / 34$ & 14527 \\
\hline 63 & $51 / 1$ & 2763 & 148 & $277 / 1$ & 77569 \\
\hline 64 & $101 / 1$ & 10513 & 151 & $283 / 157$ & 435223 \\
\hline 67 & $155 / 1$ & 24499 & 156 & $149 / 1$ & 22657 \\
\hline 73 & $79 / 10$ & 9511 & 157 & $28 / 167$ & 265813 \\
\hline
\end{tabular}


Table 2: Cyclic Cubic Fields (continuation)

\begin{tabular}{|c|c|c|c|c|c|}
\hline$h$ & $m$ & $\mathfrak{f}$ & $h$ & $m$ & $\mathfrak{f}$ \\
\hline 163 & $20 / 119$ & 134989 & 256 & $427 / 237$ & 991447 \\
\hline 169 & $212 / 1$ & 45589 & 259 & $364 / 1$ & 133597 \\
\hline 171 & $65 / 6$ & 5719 & 268 & $472 / 1$ & 224209 \\
\hline 172 & $169 / 36$ & 58477 & 271 & $869 / 582$ & 5320951 \\
\hline 175 & $254 / 1$ & 65287 & 273 & 198/1 & 39807 \\
\hline 181 & $446 / 139$ & 558787 & 277 & $367 / 1$ & 135799 \\
\hline 183 & 196/1 & 39013 & 279 & $822 / 145$ & 45277 \\
\hline 189 & $119 / 8$ & 17593 & 283 & $163 / 115$ & 201829 \\
\hline 192 & $311 / 121$ & 48769 & 289 & & \\
\hline 193 & $403 / 1$ & 163627 & 291 & $58 / 299$ & 122857 \\
\hline 196 & $20 / 121$ & 139429 & 292 & $359 / 1$ & 129967 \\
\hline 199 & $262 / 257$ & 865087 & 300 & $171 / 1$ & 29763 \\
\hline 201 & $207 / 1$ & 43479 & 301 & $70 / 99$ & 113899 \\
\hline 208 & $290 / 1$ & 84979 & 304 & $406 / 1$ & 166063 \\
\hline 211 & $688 / 149$ & 980689 & 307 & $295 / 337$ & 1407391 \\
\hline 217 & $259 / 1$ & 67867 & 309 & $330 / 1$ & 109899 \\
\hline 219 & $211 / 1$ & 45163 & 313 & $463 / 1$ & 215767 \\
\hline 223 & $206 / 1$ & 43063 & 316 & $395 / 1$ & 157219 \\
\hline 225 & $233 / 1$ & 54997 & 324 & $89 / 36$ & 29197 \\
\hline 228 & $59 / 6$ & 4867 & 325 & $343 / 1$ & 118687 \\
\hline 229 & $304 / 1$ & 93337 & 327 & $119 / 12$ & 19741 \\
\hline 237 & $44 / 215$ & 63763 & 331 & $5 / 108$ & 106621 \\
\hline 241 & $136 / 167$ & 337633 & 333 & $39 / 185$ & 47313 \\
\hline 243 & $81 / 94$ & 15561 & 336 & $707 / 348$ & 29467 \\
\hline 244 & $332 / 1$ & 111229 & 337 & & \\
\hline 247 & $368 / 1$ & 136537 & 343 & $266 / 1$ & 71563 \\
\hline 252 & $34 / 155$ & 33313 & 349 & $131 / 12$ & 23173 \\
\hline
\end{tabular}


Table 3: Cyclic Quintic Fields

\begin{tabular}{|c|c|c|}
\hline$h$ & $g(X)$ & $\mathfrak{f}$ \\
\hline 1 & $X^{5}+X^{4}-4 X^{3}-3 X^{2}+3 X+1$ & 11 \\
\hline 5 & $X^{5}-65 X^{4}-6395 X^{3}+7840 X^{2}-1625 X+1$ & 275 \\
\hline 11 & $X^{5}+4 X^{4}-70 X^{3}+135 X^{2}+54 X+1$ & 191 \\
\hline 16 & $X^{5}+16 X^{4}-274 X^{3}+817 X^{2}+178 X+1$ & 941 \\
\hline 25 & $X^{5}+1640 X^{4}+41950 X^{3}-7043 X^{2}-15122 X+1$ & 2651 \\
\hline 31 & $X^{5}+X^{4}-4912 X^{3}-32913 X^{2}+4053123 X+17302471$ & 12281 \\
\hline 41 & $X^{5}+X^{4}-4024 X^{3}-73244 X^{2}+1163776 X+5996224$ & 10061 \\
\hline 55 & $X^{5}+49 X^{4}+452 X^{3}+1125 X^{2}-207 X+1$ & 1271 \\
\hline 61 & $X^{5}+X^{4}-8560 X^{3}+255100 X^{2}+1951600 X-5058176$ & 21401 \\
\hline 71 & $\begin{array}{r}X^{5}+X^{4}-33388 X^{3}-1459073 X^{2}+31681585 X \\
+1537601101\end{array}$ & 83471 \\
\hline 80 & $X^{5}+25 X^{4}-460 X^{3}+1605 X^{2}+285 X+1$ & 1775 \\
\hline 81 & $\begin{aligned} X^{5}+524 X^{4}-558634 X^{3}+ & 87180396 X^{2} \\
& +1430815089 X-443695552\end{aligned}$ & 1671161 \\
\hline 101 & Not found. & $\longrightarrow$ \\
\hline 121 & $X^{5}+X^{4}-5728 X^{3}+7447 X^{2}+7652455 X-3749609$ & 14321 \\
\hline 125 & $X^{5}+X^{4}-5592 X^{3}+32436 X^{2}+5992704 X-2659392$ & 13981 \\
\hline 131 & Not found. & - \\
\hline 151 & $\begin{array}{r}X^{5}+X^{4}-150036 X^{3}+30802473 X^{2}-2034901683 X \\
+34977429477\end{array}$ & 375091 \\
\hline 155 & $X^{5}-1755 X^{4}+98729 X^{3}-101785 X^{2}+3278 X+1$ & 8651 \\
\hline 176 & $X^{5}+X^{4}-5068 X^{3}+158641 X^{2}-847031 X-4729247$ & 12671 \\
\hline 181 & Not found. & - \\
\hline 191 & $X^{5}+X^{4}-8844 X^{3}-72524 X^{2}+5838896 X-42516736$ & 22111 \\
\hline
\end{tabular}




\section{REFERENCES}

[1] H. Cohen, Advanced topics in computational number theory, Springer-Verlag, New York, 2000.

[2] G. Cornell, Group theory and the class group, Number theory and applications, NATO adv. Sci. Inst. Ser. C, 265 (1989), 347-352.

[3] G. Cornell and M. Rosen, Group-theoretic constraints on the structure of the class group, J. Number Theory, 13 (1981), 1-11.

[4] R. Dentzer,, Polynomials with cyclic Galois group, Comm. in Algebra, 23 (1995), 1593-1603.

[5] K. Iwasawa, A note on ideal class groups, Nagoya Math. J., 27 (1966), 239-247.

[6] E. Lehmer, Connection between Gaussian period and cyclic units, Math Comp., 50 (1988), 535-541.

[7] J. M. Masley, Class numbers of real cyclic number fields with small conductor, Compositio Math., 37 (1978), 297-319.

[8] J-P. Serre, Topics in Galois theory, Jones and Bartlett Publishers, Boston, 1992.

[9] L. C. Washington, The non-p-part of the class number in a cyclotomic $\mathbb{Z}_{p}$-extension, Invent. Math., 49 (1978), 87-97.

[10] L. C. Washington, Introduction to cyclotomic fields, 2nd edition, Springer-Verlag, New York, 1997.

\section{Toru Komatsu}

Department of Mathematics

Tokyo Metropolitan University

Hachioji, Tokyo 192-0397

Japan

trkomatu@comp.metro-u.ac.jp

Shin Nakano

Department of Mathematics

Gakushuin University

Toshima-ku, Tokyo 171-8588

Japan

shin@math.gakushuin.ac.jp 